\title{
Detection and Demining of Landmines using Artificial Intelligence
}

\author{
Lekshma $\mathbf{N}^{1}$, Samyuktha $\mathbf{P}^{2}$, Swedha $\mathbf{P}^{3}$, Vigneswari $\mathbf{P}^{4}$ \\ B.E - ECE, Avinashilingam Institute for Home Science and Higher Education for Women, \\ School of Engineering, Coimbatore, Tamilnadu ${ }^{1,2,3}$ \\ Assistant Professor, Avinashilingam Institute for Home Science and Higher Education for Women, \\ School of Engineering, Coimbatore, Tamilnadu ${ }^{4}$
}

\begin{abstract}
The main objective of this project is to design a low cost, wireless ground vehicle which identifies landmine and to demine them, without human interference, using Artificial Intelligence. This detector will wirelessly communicate with a server to transmit the detected information such as the location of the metal, object and the captured images of the landmine. The proposed system will help in significantly reducing the risk posed to the human detectors life while they are in a field of mine. Besides, automation will guarantee an easier and fast scanning process that will open the door to more possibilities such as automatically investigating the received bit sizes of data for recognizing mine from other left behind normal objects. In addition, Artificial Intelligence is used which reduces the rate of false alarm.
\end{abstract}

Keywords: Wireless, ground vehicle, Artificial Intelligence, images.

\section{INTRODUCTION}

The landmines are weapons developed to be deployed near or under the ground to explode when it is contacted by a person or a vehicle. Basically landmines can be divided into two : AP anti-personnel and AT anti-tank landmines, depending on the target and the cost. Demining can be classified into two depending on the problem definition. The military minesweeping: to achieve fast clearing with acceptable reliability to open a safe passage for military personals to enter their battlefield. Humanitarian demining to achieve $100 \%$ clearance of the ground at acceptable cost. This project is to design a low cost, wireless controlled ground vehicle which identifies landmine and to demine them, without human interference, using Artificial Intelligence.

\section{LITERATURE SURVEY}

2.1 Nikhil VasudevNambiar, KaurGurpreet (2017), proposed a paper on the title 'Automatic Landmine Detection by Pattern Trace', which presents a novel approach for auto detection of Landmine in Ground Penetrating Radar (GPR) data using a machine learning technique. The limitations of the paper wereassuming probability value for adaptive threshold which may vary on basis of GPR used. [1]

2.2KaurGurpreet (2016), proposed a paper on the title 'Multi algorithm-based Landmine Detection using Ground Penetration Radar', paper deals with analyzing the GPR data, to detect anti-tank landmines buried in various conditions. The sigma values may vary when tested in the actual scenarios. [2]

2.3IhabMakki, RaficYounes (2017), proposed a paper on the title 'Classification algorithms for landmine detection using hyper spectral imaging', presents some preliminary results obtained when applying various supervised classification algorithms for the detection of landmines in hyper spectral images. The detection performance of the algorithms in the case of a multi-target scenario must be improved. [3]

2.4 Rajesh KannanMegalingam, VamsiGontu, RuthvikChanda, Prasant Kumar Yadav, AlladaPhanindra Kumar (2017), proposed a paper on the title 'Landmine Detection and Reporting using Light Weight ZumoBot', proposes a simple bot to detect landmines and mark their locations using autonomous navigation using a simple Zumo32U4 robot but the rate of false alarms are high. [4]

2.5 Ibrahim A. Hameed(2016), proposed a paper on the title 'Motion Planning for Autonomous Landmine Detection and Clearance Robots', a motion-planning algorithm to enable landmine detection and clearing robots to systematically scan a minefield, detect landmines and clear is presented.Large amount of data requires better algorithm to achieve real time landmine detection is the limitation of this proposed system. [5] 


\section{International Advanced Research Journal in Science, Engineering and Technology}

Vol. 8, Issue 6, June 2021

\section{DOI: $10.17148 /$ IARJSET.2021.8648}

2.6Xinghua Shi, Dandancheng, Zhiyan Song, Chenghao Wang (2018), proposed a real-time landmine detection method used on a 3-dimensional (3-D) dataset of vehicle array GPR is presented on the title 'A Real-time Method for Landmine Detection Using Vehicle Array GPR'.This approach can detect landmines only for 5kms in an hour. [6] 2.7Khaoula TBARKI, Salma BENSAID, Riadh KSANTINI, Zied LACHIRI (2016) proposed a detection method based on support vector machine (SVM) using one-dimensional GPR delivered data called A scans is proposed on the topic 'RBF Kernel based SVM classification for landmine detection and discrimination'. The limitations are that it cannot discriminate between landmines and false alarms. [7]

\section{EXISTING SYSTEM}

A lot of research has been already done in the domain of mine detection, extracting and demining. sensor technology, Ground Penetrating Radar (GPR), electromagnetic induction are the areas where the research has been done. For all these technologies, detection is most probably done by expert humans (detectors) holding a detector to scan a suspected area of land. This process introduces a high cost in terms of time, money and man power.

\section{PROPOSED SYSTEM}

With reference to the above-mentioned papers, some of the architectures designed for the detection of landmine will be adopted in this system. With the help of artificial intelligence, the rate of false alarm will be decreased.

\section{COMPONENTS REQUIRED}

- Inductive Proximity sensor (RM 18)

- Ultrasonic sensor (HC-SR04):

- Raspberry Pi 4 Model B

- LCD display

- ARM Motor

- DC relay switch

- Driver

\section{BLOCK DIAGRAM}

TRANSMITTER MODULE

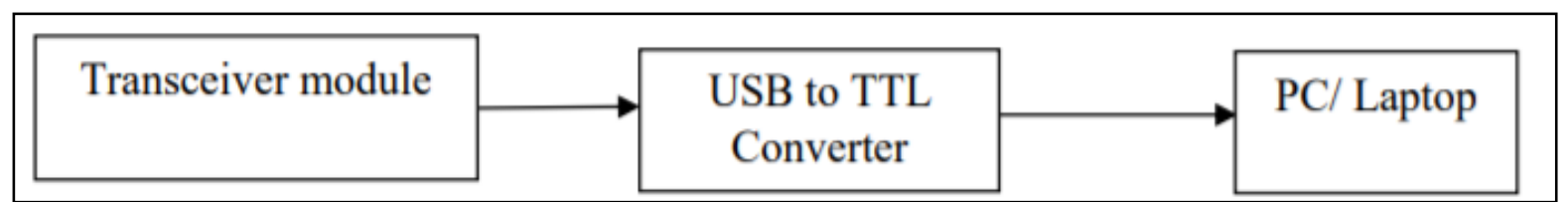

Fig 6.1 Block diagram of the Transmitter module

\section{RECEIVER MODULE}


International Advanced Research Journal in Science, Engineering and Technology

Vol. 8, Issue 6, June 2021

DOI: $10.17148 /$ IARJSET.2021.8648

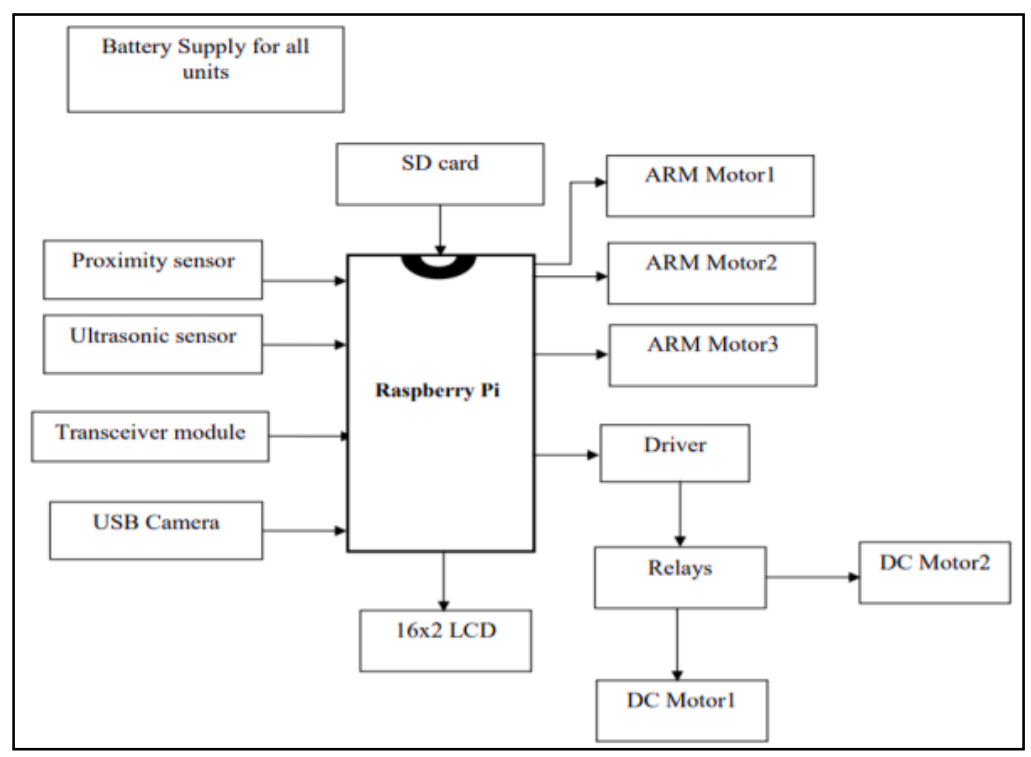

Fig 6.2 Block diagram of the receiver module

\section{FLOWCHART}

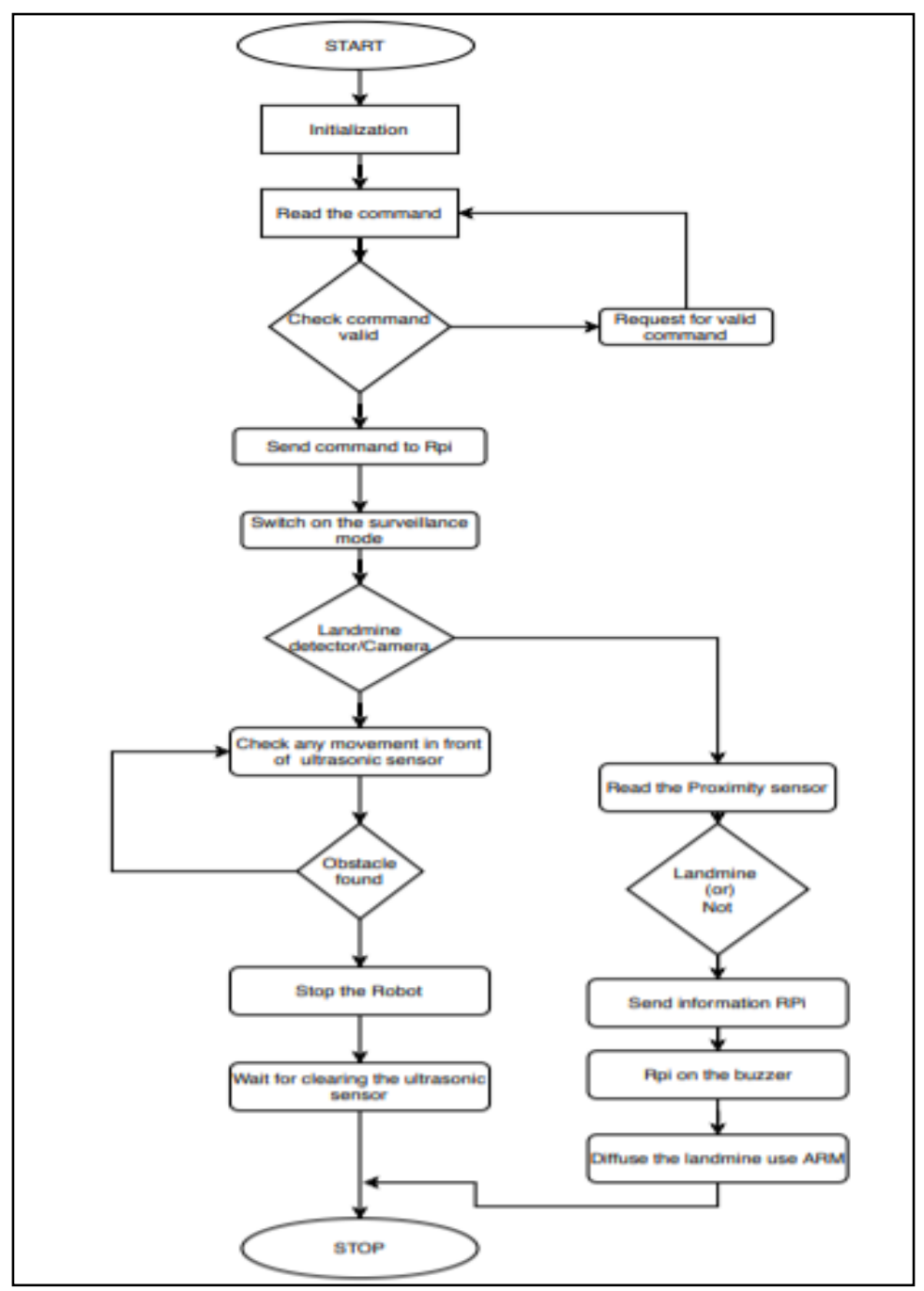

Fig 7.1 Flowchart 


\section{International Advanced Research Journal in Science, Engineering and Technology}

Vol. 8, Issue 6, June 2021

DOI: $10.17148 / I A R J S E T .2021 .8648$

\section{WORKING}

This detector is based on a Raspberry Pi minicomputer ship that will act as the brain of a small robot that will act as the brain of a small robot to automatically detect and transfer the information about any found metal object to the central unit.The ground vehicle activates when a valid command is given. When it switches to surveillance mode, it reads the proximity sensor and if any metal is detected the information is sent to RPi which controls the input and output, which in turn starts the buzzer. Then the landmine is diffused using the robotic arm. The ultrasonic sensor is used to find if any obstacle is in the path of the ground vehicle. If there is any such obstacle, the ground vehicle waits for the path to be cleared.

\section{SPECIFICATION}

Inductive Proximity sensor (RM 18): It is used for positioning and detecting the metal objects.

Ultrasonic sensor (HC-SR04): It is used to detect the obstacles on the path of the robot.

Raspberry Pi 4 Model B: The Raspberry Pi has the ability to interact with the outside world and it acts as a mini computer.

LCD display: It is used to display the commands.

ARM Motor: Angular movements of joints of the robots are needed to be controlled, which can be achieved using servo motors.

DC relay switch: It is used for moving the ground vehicle.

Driver: A driver is a circuit or component used to control another circuit or component, such as a highpower transistor, liquid crystal display (LCD), and numerous others.

\section{HARDWARE DESCRIPTION}

Fig 10.1 shows the hardware setup of the proposed system. The detection process that is used is based on a metal detector that can detect metal objects even with no direct contact with them. Tests have been done to detect metal objects on different area of lands and the detector could transfer the required information such as the exact location of the objects and the images of the area where the object was found with very good precision. The proposed Robo-Pi unit can be considered as a prototype that still needs real tests to show its efficiency.

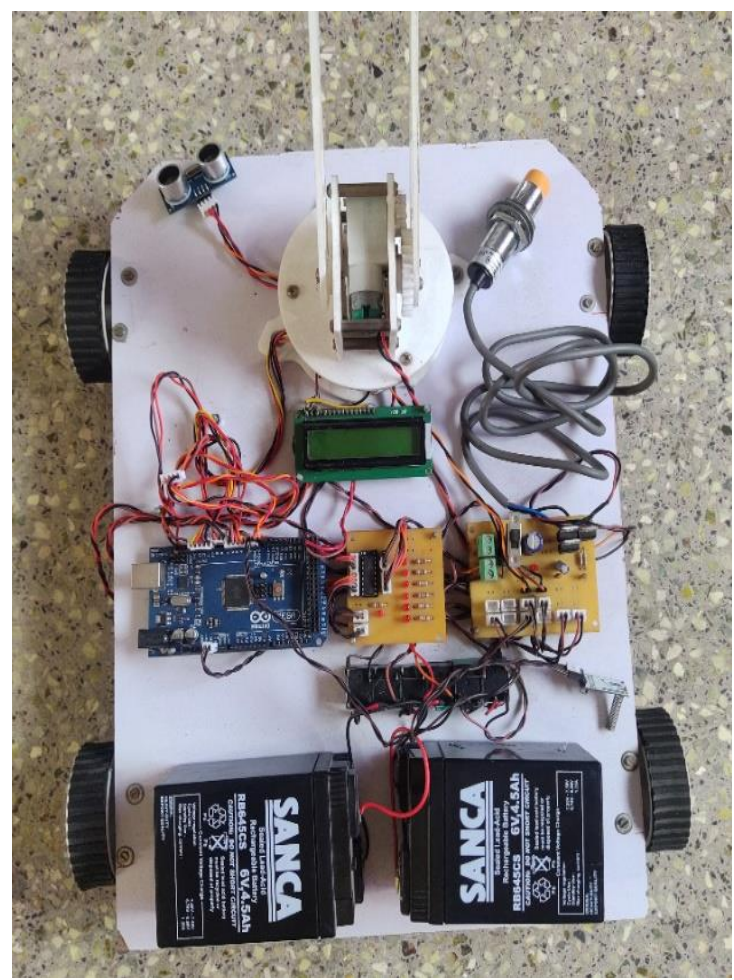

Fig 10.1 Hardware setup of the proposed system 


\section{International Advanced Research Journal in Science, Engineering and Technology}

Vol. 8, Issue 6, June 2021

DOI: $10.17148 /$ IARJSET.2021.8648

\section{CONCLUSION}

The objective of this project is to design and implement an efficient low-cost and automatic landmine detector. The detector is based on a raspberry pi minicomputer ship that will act as a brain of a small robot to automatically detect and transfer the information about any found metal object to a central unit that will later be responsible of investigating the received data to benefit from it in determining the location and maybe the danger of the found object and hence, find a way to extract or disable it. The future scope of this proposed system includes the use of artificial intelligence to reduce the rate of false alarms by differentiating the landmines from other metal scraps.

\section{REFERENCES}

[1]. Rajesh KannanMegalingam, VamsiGontu, RuthvikChanda, Prasant Kumar Yadav, AlladaPhanindra Kumar, 'Landmine Detection and Reporting using Light Weight ZumoBot' published on September 2017.

[2]. Nikhil VasudevNambiar, KaurGurpreet (2017), 'Automatic Landmine Detection by Pattern Trace' published on January 2017.

[3]. KaurGurpreet, 'Multi algorithm based Landmine Detection using Ground Penetration Radar' published on May 2016.

[4]. IhabMakki, RaficYounes, 'Classification algorithms for landmine detection using hyper spectral imaging' published on May 2017.

[5]. Xinghua Shi, Dandancheng, Zhiyan Song, Chenghao Wang, 'A Real-time Method for Landmine Detection Using Vehicle Array GPR' published on May 2018

[6]. Zoran Najdovski, Christopher Mawson, Hieu Trinh, Saeid Nahavandi, 'Solution to Robotic Landmine Detection through use of path planning and Motor Control' published on June 2006

[7]. Khaoula TBARKI, Salma BENSAID, Riadh KSANTINI, Zied LACHIRI, 'RBF Kernel based SVM classification for landmine detection and discrimination' published on 2016.

[8]. Varshaa a; Vinitha V; Usha Nandhini D; Yogeshwaran R; Soundharya B M. "Artificial intelligence and its applications- A Review". International Research Journal on Advanced Science Hub, 1, 2, 2020, 1-4. doi: 10.47392/irjash.2019.11

[9]. Suneetha V; Salini Suresh; Niharika Sinha; Sabyasachi Prusty; Syed Jamal J. "Enhancement in the World of Artificial Intelligence". International Research Journal on Advanced Science Hub, 2, Special Issue ICARD 2020, 2020, 276-280. doi: 10.47392/irjash.2020.132

[10]. Y.Sun \& J.Li, 'Time-frequency analysis for plastic landmine detection via forward-looking ground penetrating Radar' published on Aug 2003.

[11]. Nikhil VasudevNambiar, KaurGurpreet, 'Automatic Landmine Detection by Pattern Trace' published on January 2017.

[12]. Maki K.Habib, 'Humantarian Demining Mine Detection and Sensors’ Industrial Electronics (ISIE), 2011 IEEE International Symposium on 2730 June 2011 in Gdansk, Poland. 